\title{
A Refined Approach of Image Retrieval Using RBF-SVM Classifier
}

\author{
Mohd. Aquib Ansari ${ }^{1}$ and Manish Dixit ${ }^{2}$ \\ ${ }^{1,2}$ Dept. of CSE/IT \\ 1, 2 Madhav Institute Technology \& Science, \\ ${ }^{1,2}$ Gwalior, India \\ aaquib.mits@gmail.com,dixitmits@gmail.com
}

\begin{abstract}
In recent trends, Content Based image retrieval (CBIR) is a vast as well as an attractive research area, where various active researchers are doing their respective work. It is the widely used technique of image retrieval, which enables the system to retrieve the relevant images from wide range of image database following the user's query image. In designing of good CBIR system, to select an appropriate feature descriptor which can represent the image content efficiently is not an easy task. In this proposed work, an efficient CBIR system has been suggested for image retrieval which mainly focused on the classification of image database as well as appropriate image representation. Here, we used HSV histogram, discrete wavelet transform and local as well as global edge histogram descriptor with SVM classifier based on RBF kernel function. In the scheme of feature evaluation, first extracted quantized HSV histogram to extract the color feature of image, applied discrete wavelet transform on the each component (Hue, Saturation and value components) of the HSV image to extract the complex texture pattern of image and for evaluation of geometric as well as spatial information of image applied local as well as global edge histogram descriptor on the Value component of HSV Image. After combining the all feature vectors, SVM Classifier applied on the image feature database to classify the images appropriately. For experimental analysis, this proposed methodology applied on the Wang image database of 1-k images with 10 categories. On the basis of Precision, recall and accuracy, it is found that this system, based on classification, is performing well in comparison to other existing proposed schemes.
\end{abstract}

Keywords: CBIR; HSV histogram quantization; discrete wavelet transform; edge histogram descriptor; RBF-SVM classifier

\section{Introduction}

Digital images contain the well-known as well as essential information which is used widely in the various regions like bio-medical, military, journalism, space observation etc. [1]. These popular areas are producing digital images day by day in a large amount. It is become more tedious to retrieve or search these user's perspective image from the large image repository. In order to manage these circumstances, a huge database for storing and managing these images and an efficient image retrieval system for retrieving the essential images according to user's need must be provided in these regions. Now, retrieving the user's perspective images accurately is an important as well as the challenging task for the various researchers. Thus, content based image retrieval (CBIR) is discovered in 90's to fulfil these requirements. CBIR is a technique of image retrieval and an application of computer vision [2]. It uses the visual contents of digital image to extract the features and

Received (January 9, 2017), Review Result (April 26, 2017), Accepted (May 5, 2017) 
on the basis of these features, it browses or retrieves the images from the large image repository following the user's input image. These visual contents such as texture, color and shape are also be called as low level or pixel level feature of the image. These pixel level features are extracted from the each image of image repository and stored in the feature database. The same thing is happened for the user's query image and construct the vector for the query image also. Then, similarity measurement is performed for comparative evaluation between the query image feature and image database feature to get accurate images that are similar to query image pattern. The basic diagram of CBIR is presented in Figure 1.

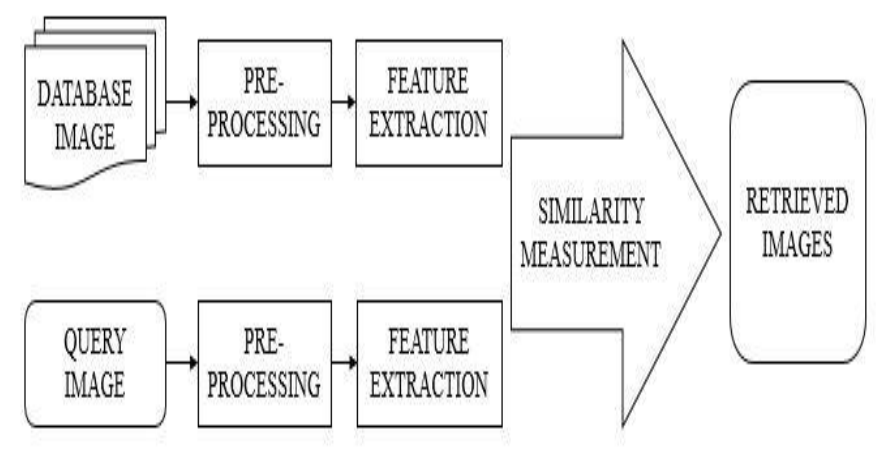

Figure 1. Basic Diagram of CBIR

Here, Pre-processing [3] step involves resizing of image, conversion of image spaces for further evaluation, filtering (to remove noise from image), normalization and segmentation. It is an important step in CBIR. The feature extraction step in CBIR extract the features of the image in the form of numerical array which can represent the whole single image. The next and essential step of CBIR is similarity measurement, which matches the query image with the each image of image repository and gives result as visually similar images following to query image pattern.

\section{Related Work}

In 2016, Sawet Somnugpong and Kanokwan Khiewwan [4] proposed an efficient algorithm for CBIR system. This combine approach used color and geometric descriptor to extract the features of the image. In this paper, Color Correlograms to extract the spatial color information of an image and Edge Direction Histogram to extract the geometric information of the edges present in an image are used. This combined approach produced the good results in term of precision and recall over the existing system.

In 2015, Katta Sugamya, Suresh Pabboju and Dr. A. Vinaya Babu [5] proposed an efficient retrieval system by using color, texture and shape descriptor. They used color auto correlogram as a color feature to extract the color information of the image, gabor wavelet and wavelet transform to extract texture and shape information of the image. On the combination of these feature, an efficient retrieval system is developed. In the next step, to removes the noisy images and to improve the accuracy of CBIR system, they employed the SVM classification method on this fused approach and find that the generated confusion matrix successfully separated the relevant and irrelevant images.

In 2015, Ekta Gupta and Dr. Rajendra Singh Kushwaha [6] developed an efficient CBIR system by using global as well local feature using discrete wavelet transform and support vector machine. This fused CBIR method based on classification algorithm touched the average accuracy of 82 percent and also proved that it is working well in precision and recall. 
In 2016, S. Sankar Ganesh and K. Ramar [7] proposed a novel approach of image retrieval based on heuristic particle swarm optimization. In this approach, the value of mean and standard deviation are used to extract the color feature of image and active contour is used to extract the region feature involved in image. Here, PSO is applied on the retrieval system to decrease the semantic gap between the results and user need. This approach is performing well in precision and reducing performing complexity also.

In 2014, Dr. Ananthi Sheshasaayee and Jasmine .C [8] surveyed on various relevance feedback techniques and found that the semantic gap as well as performance problem can be reduced by the relevance feedback mechanism. They performed the comparative study on various relevance feedback techniques based on their performance, issues and also described their advantages and limitation in brief. They also explained that how a relevance feedback mechanism puts a significance role in the region of content based image retrieval.

In 2014, Vinne. V. Kawade and Arti. V. Bang [9] introduced a retrieval system based on user's perspective. This interactive CBIR system involved several features of color like mean, standard deviation and bitmap as well as the feature of texture like edge histogram algorithm and GLCM to extract the efficient color as well as texture features of an image. On the fusion of these color and texture feature, the genetic algorithm is applied over it which benefitted to reduce the semantic gap between the user's expectation and the retrieval results. In this paper, the experimental results showed that this system improved so well after applying the genetic algorithm.

SVM is the popular classification technique of machine learning due to its attractiveness and well performance behavior. It was developed by Vapnik [10]. SVM classifies the images more accurately than other classification techniques and mainly used to solve the real world problems like object recognition, voice recognition, digital handwriting recognition, data classification etc.

\section{Proposed Methodology}

This proposed approach of CBIR system involved HSV color histogram, discrete wavelet transform, global as well as local histogram descriptor and SVM Classifier based on RBF kernel function. The overall proposed CBIR system is presented in Figure 2.

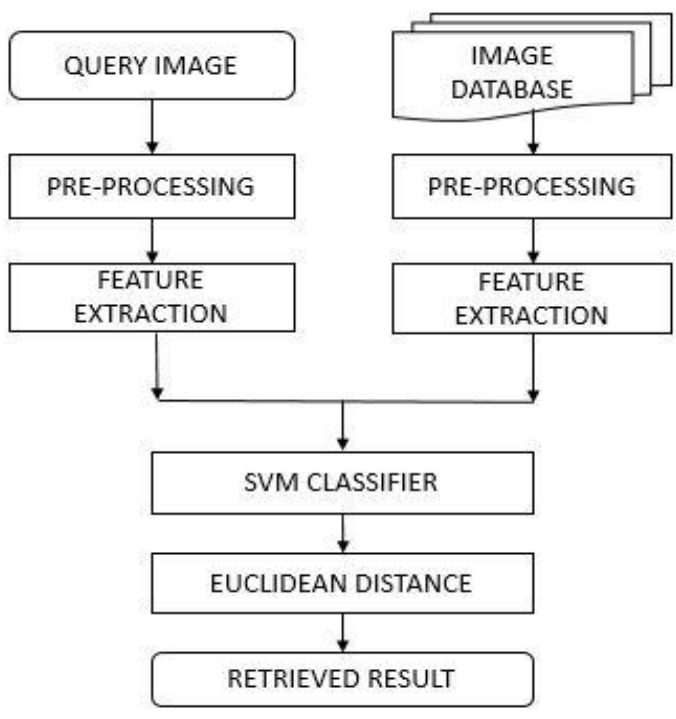

Figure 2. Proposed System 
CBIR system extracts the visual features such as color, texture and shape (in manner to illustrated in Figure 3) from the each image of the image repository one by one and stored these feature vector in the different database, called feature database. This feature database represents the all image of image database uniquely. The user enters the image as query image to the system and now, this system extracts the same visual features of the image and form a query image feature vector. Then, performed the classification algorithm Support Vector Machine on these feature vectors. Which successfully classified the images according to its training and testing feature vectors with the presence of kernel function. Here, the confusion matrix is obtained as a result to describe the system performance [11]. Along with SVM classifier, the Euclidean distance is used to find most similar images corresponding to query image. The flow diagram of feature extraction is illustrated in Figure 3.

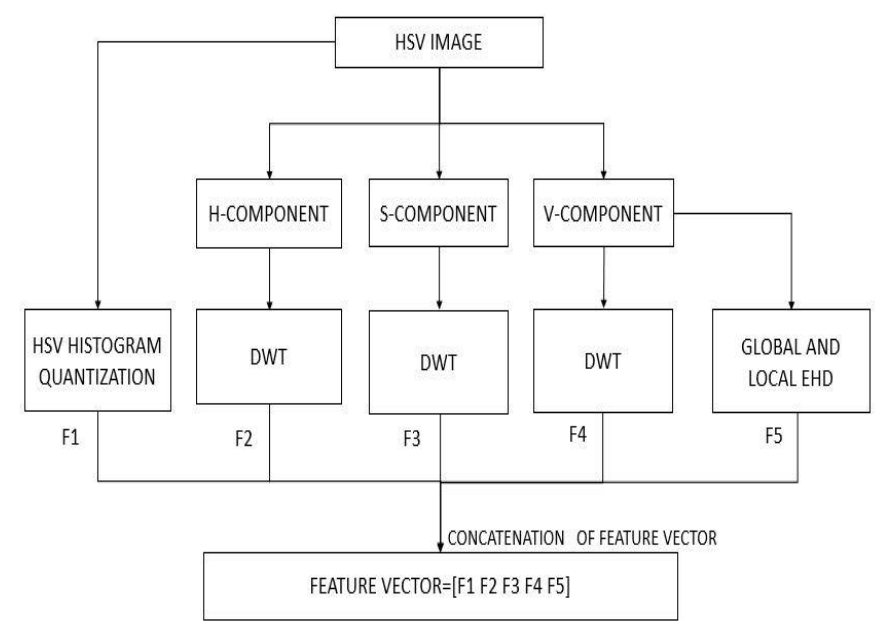

Figure 3. Feature Extraction Scheme

The involved techniques for feature extraction are as follows.

\section{A. HSV Color Histogram Quantization}

In this paper, we used HSV color histogram quantization [12] as color descriptor to extract the color information of an image. An image contains the color contents which represent the image visually. The color histogram gives the frequency of color contents occurred in an image. In HSV color histogram, the distribution of gray levels or intensity values with in an image for each bin is evaluated. These bins show that how many times the particular color is involved with in an image. Sometimes the large number of bins can't reflect the whole image effectively because of not only the large number of bins will increase the computational cost but also it will not be good for efficient indexing for the image database. Then, to overcome this problem we quantized the number of bins value so that the color distribution of histogram takes place effectively within less number of bins. Here, we quantized the global 256 bins into 72 bins (for $\mathrm{H} 8$ bins, for saturation 3 bins and for value 3 bins $=8 \times 3 \times 3$, total bins $=72$ bins). Quantization process depends on thresholding [13]. The quantized threshold value for hue, saturation and value are as follows: 


$$
\text { Hue }(H)=\left\{\begin{array}{cl}
0, & H \in[315,360] \\
1, & H \in[0,25] \\
2, & H \in[26,40] \\
3, & H \in[41,120] \\
4, & H \in[121,190] \\
5, & H \in[191,270] \\
6, & H \in[271,295] \\
7, & H \in[296,314] \\
\text { Saturation (S), Value }(V)= \begin{cases}0, & (S, V) \in[0,0.2] \\
1, & (S, V) \in[0,2,0.7] \\
2, & (S, V) \in[0.7,1]\end{cases}
\end{array}\right.
$$

This quantization based HSV color histogram gave less number of feature vector which represents an image uniquely and efficiently.

\section{B. Discrete Wavelet Transform}

Texture is widely used feature, which describes the structure information of surface. Here, we used discrete wavelet transform [14] to extract the texture information from the surface of the image. The wavelet is the small waves of fluctuating frequency with the finite duration. The discrete wavelet transform transforms the spatial domain signal into frequency domain. The concept behind wavelet transform is that a small size and low contrast object can represent the object of bigger size at high resolution. DWT discovers the efficient knowledge from the different scales of the signal by passing it through low as well as high pass filters. The wavelet transform is a multiresolution approach means that the information which can't be identified at one resolution, it can be identified at another resolution. So, it is its biggest advantage. In Figure 4, the two-level discrete wavelet transform is illustrated. Here, the signal is decomposed into LL, HH, HL, LH bands by applying the DWT on it. The LL, HH, HL and LH frequency bands contain the approximation, diagonal, horizontal and vertical details of the signal. The LL band is decomposed again into LL1, LH1, HL1 and HH1 [15]. Here, we used two level discrete wavelet transform for evaluating the texture information of image.

\begin{tabular}{|c|c|c|}
\hline LL 1 & HL 1 & \multirow{2}{*}{ HL } \\
\hline LH 1 & HH 1 & \\
\hline LH & HH \\
& & \\
\hline
\end{tabular}

Figure 4. Wavelet Decomposition

\section{Edge Histogram Descriptor}

The shape can be defined as the portion of the space which is engaged by the object. The shape feature is a significant feature to define the shape information of object and it is used widely. Here, we used edge histogram descriptor (EDH) to extract the spatial information from the image. EHD is a MPEG-7 family descriptor which is used to evaluate the different type of orientation of edges present in an image. To calculate the edge information of the image, first, need to partition the digital image into $4 \times 4$ subimages. Then, each sub-image is further partitioned into non-overlapping square-blocks called image blocks. Where size of the image block is dependent on the resolution of the 
image. From these image blocks, the different types of edges are extracted on the basis of filter coefficient according to algorithm of EHD [16]. The various orientations of edge [4] are vertical, horizontal, 45-degree, 135 degree and no-direction edges, which are shown in Figure 5. From the each sub-image, five bins of edges histogram are extracted. By normalizing the number of image blocks present in each sub-image, the value of each bin of particular sub-image is evaluated. At last, all these normalized values of bins are collected and form a feature vector which holds the spatial information of image. Here, we used local as well as global EHD to make this descriptor more effective. The total bins for local EHD are 80 bins and for Global EHD are 5 bins.

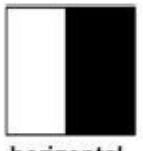

horizontal

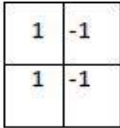

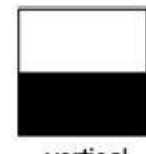

vertical

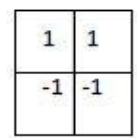

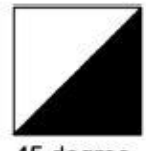

45 degree

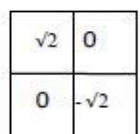

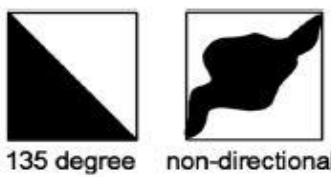

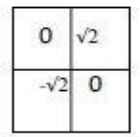

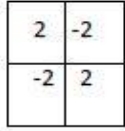

Figure 5. Edge Type with Its Mask

\section{SVM Classifier}

Support vector machine [6] [11] is a classification algorithm based on supervise learning that is used here to classify the digital images into separate classes by the help of hyper plain. SVM gives a decent way to find the surface which can separate the classes. The basic objective of SVM classifier is to find the optimal separating hyper plain which maximizes the margin of training data. The optimal hyper plane would have the maximum margin to achieve minimum noise. None of the training data just fall within the margin. The basic idea of classification through SVM is illustrated in Figure 6.

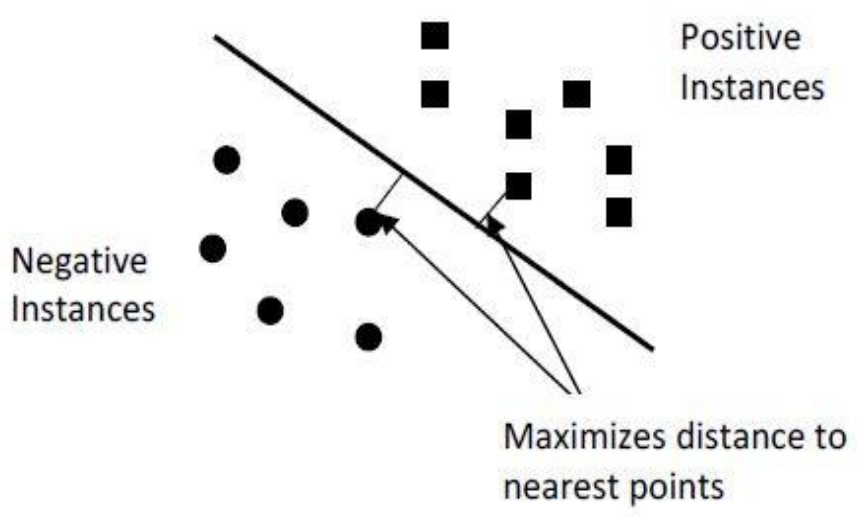

Figure 6. SVM Classification

In this Figure 6, this is the case of two dimensions with the linearly separable dataset. SVM classified the dataset into two instances such as negative and positive instances. Here, a hyper plane is identified which is separating the dataset into two class with maximum margins. The points or instances which fall on the margins are called support vectors [6].

The mathematical evaluation of SVM [17] is given as follows:

$$
\begin{gathered}
\min _{b_{i},} \frac{1}{2}\|w\| \\
y_{m}=\bar{x}_{m} \cdot \bar{w}+b \geq+1 \rightarrow+1 \\
y_{m}=\bar{x}_{m}, \bar{w}+b \leq-1 \rightarrow-1 \\
\because \quad y_{m}\left(\bar{x}_{m}, \bar{w}+b\right) \geq \forall m
\end{gathered}
$$


Where, $y_{m}$ identifies the each data values of $x_{m}$ and gives label +1 and -1 to each data point (classifies into positive and negative instance of the class). The equation of the hyper plane is as follows:

$$
v=\bar{x} \cdot \bar{w}+b
$$

Where, $\mathrm{b}$ is bias. One of the best way to solve this constraint is to use the Lagrange theory which converts it into dual Lagrange problem. The dual problem is given as follows:

$$
\begin{gathered}
\min _{\alpha}\left(L_{D}\right)=\min _{\alpha} \frac{1}{2} \sum_{m=1}^{N} \sum_{m=1}^{N} y_{m} y_{n}\left(\bar{x}_{m} \bar{x}_{n}\right) \alpha_{m} \alpha_{n}-\sum_{m=1}^{N} \alpha_{m} \\
\sum_{m=1}^{N} \alpha_{m} y_{n}=0 \\
; \quad \alpha_{m} \geq 0, \forall m
\end{gathered}
$$

Where $\alpha_{m}$ is Lagrange Multiplier for data point $\mathrm{x}_{\mathrm{m}}$.

SVM uses the kernel function to separate the non-linear separable data. This kernel function is the part of SVM. In this paper, we used Radial Basis Function as a kernel function which classified the data more efficiently than others. It can classify the data into more than 2 class as compare to linear and polynomial kernel function [18].

\section{E. Similarity Measurement}

Similarity Measurement is used to find the similar images in the large digital image repository following the query image which is given by the user. Here, we used Euclidean distance [4] [12] [19] to find the similar images corresponding to query image.

$$
d=\sqrt{\sum_{i=1}^{k}\left(x_{i}-y_{i}\right)^{2}}
$$

Where, $d$ is a distance vector, $x_{i}$ is the feature vector of query image, $y_{i}$ is the feature vector of image in the database and $\mathrm{k}$ is the vector's dimension.

\section{F. Proposed Algorithm}

Step 1:Select the image directory where all the images are stored.

Step 2:Input RGB image (I) of dimension $\mathrm{M} \times \mathrm{N}$.

Step 3:Preprocess the image first by resizing the image into $256 \times 384$ and then convert it to HSV image from RGB image.

Step 4:Apply the HSV histogram quantization on the HSV image and form the feature vector $\mathrm{f} 1$.

Step 5:Divide the HSV color image into three separated components such as Hue, Saturation and value components.

Step 6:Apply the discrete wavelet transform on the each component of HSV image and form feature vector $\mathrm{f} 2, \mathrm{f} 3$ and $\mathrm{f} 4$.

Step 7:Apply the global as well as local edge histogram descriptor on the value component of HSV image and form feature vector $\mathrm{f5}$.

Step 8:Combine all the feature vectors and form a single vector $\mathrm{f}=[\mathrm{f} 1, \mathrm{f} 2, \mathrm{f} 3, \mathrm{f} 4, \mathrm{f} 5]$.

Step 9:Calculate feature vector $f_{i}$ for each image of image database by repeating the step ii to step viii until the last remaining image. Then stored all these feature vectors in the separate database of the system.

Step 10:Enter the query image of dimension $\mathrm{M}$ x $\mathrm{N}$ through the user interface.

Step 11: Repeat the steps from step iii to step viii to calculate the feature vector of the query image.

Step 12:After evaluating feature vector by using step 2 to step 9. These feature vectors are applied to RBF-SVM classifier which classifies the feature vectors into different classes corresponding to query image. Then, perform the similarity measurement Euclidean distance to display the retrieved results.

Step 13:Calculate the precision, recall and accuracy after applying SVM algorithm. 


\section{Experimental Results}

\section{G. Image database}

Here, Wang image database [4] [6] [20] [23] is employed for our proposed approach. The experimental analysis is done on 1-k images of Wang database with 10 categories of image classes like African, Beach, Monuments, Busses, Dinosaurs, Elephants, Flowers, Horses, Mountain and food. Each category having 100 of images. In Figure 7, the each class of images are illustrated.

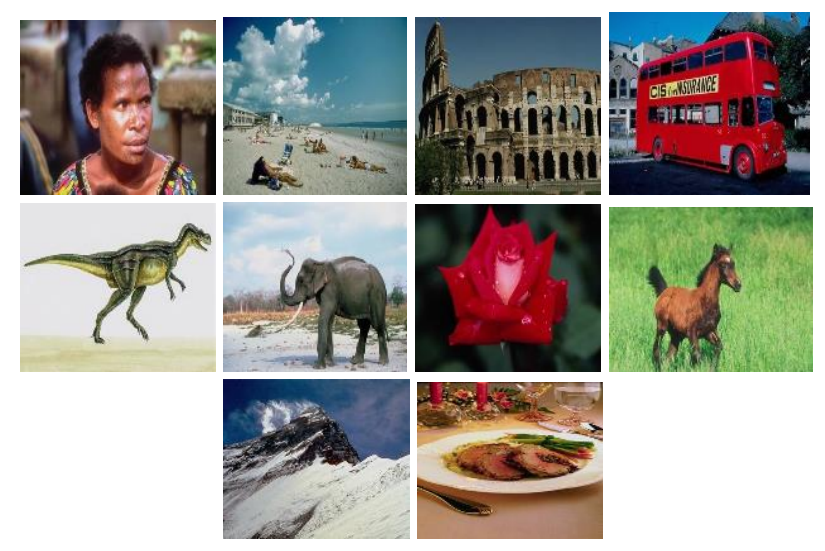

Figure 7. Classes of Wang Image Database

\section{H. Performance Evaluation}

Performance evaluation is used to evaluate the system efficiency and accuracy. Here, we measured precision, recall and accuracy rate to evaluate the performance of proposed system [21]. Where, precision and recall provides the information about effectiveness of the system. The term 'accuracy' gives the overall performance of the system.

$$
\begin{gathered}
\text { Precision }(\mathrm{P})=\frac{\mathrm{TP}}{(T P+F P)} \\
\text { Recall }(\mathrm{R})=\frac{\mathrm{TP}}{(T P+F N)} \\
\text { Accuracy }(\mathrm{A})=\frac{\mathrm{TP}+\mathrm{TN}}{(T P+F N+F P+T N)}
\end{gathered}
$$

Where, TP is True Positive, FP is False Positive, FN is False Negative and TN is True Negative.

True positive is number of rightly recognized test images. True Negative is number of rightly rejected test images. False positive is number of wrongly identified test images. False Negative is number of wrongly rejected test images.

\section{Result Analysis}

Here, Matlab R2013b tool is used for performing the experiments. The percentage of correct and incorrect classification can be derived by the confusion matrix. In Figure 8, the confusion matrix is illustrated for the predictive query image of class 1 with accuracy of 84 percent by using Manhattan distance. 


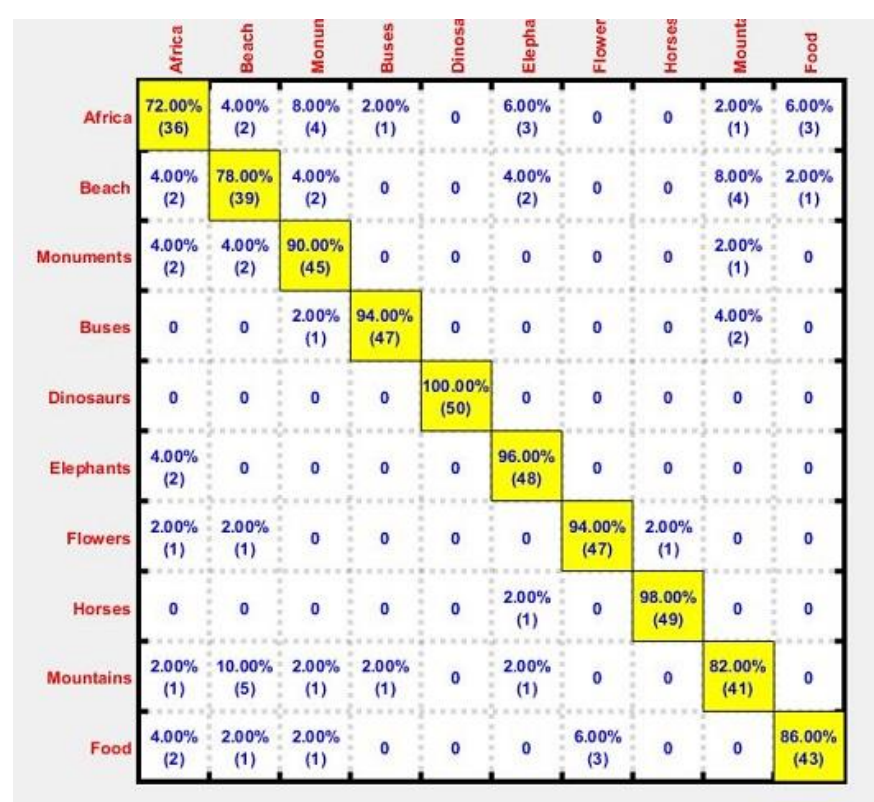

\section{Figure 8. Confusion Matrix for Predictive Query Image Class 1}

Here, we took 5 images randomly from each class as the query image and applied these images one by one to the proposed method. Then evaluated the precision, recall and accuracy for each of them. Then after, average the values of precision, recall and accuracy for the each class to calculate the average precision, average recall and average accuracy. This proposed system has evaluated the enhanced results which are shown in Table 1 . The average accuracy, evaluated by SVM for predictive query image of each class with Manhattan, Euclidean, City-Block and Relative deviation measurement, are shown in Table 1. From the result analysis, it found that the proposed system gave the good result for four similarity measurements. But for Euclidean measurement, it gave slightly more good result.

\section{Table 1. Average Accuracy with Similarity Measures}

\begin{tabular}{|l|l|l|l|l|}
\hline \multirow{2}{*}{$\begin{array}{l}\text { Image } \\
\text { category }\end{array}$} & \multicolumn{4}{|c|}{ Similarity Measurement } \\
\cline { 2 - 5 } & Manhattan & Euclidean & $\begin{array}{l}\text { City } \\
\text { Block }\end{array}$ & $\begin{array}{l}\text { Relative } \\
\text { Deviation }\end{array}$ \\
\hline African & 85.28 & 84.84 & 83.68 & 81.80 \\
\hline Beach & 85.04 & 85.84 & 84.02 & 84.60 \\
\hline Monuments & 84.88 & 88.04 & 84.88 & 86.74 \\
\hline Buses & 85.08 & 85.36 & 84.24 & 84.68 \\
\hline Dinosaurs & 84.6 & 84.76 & 84.92 & 85.02 \\
\hline Elephants & 84.16 & 84.32 & 84.08 & 82.62 \\
\hline Flowers & 84.32 & 85.58 & 84.24 & 84.00 \\
\hline Horses & 84.8 & 84.8 & 84.68 & 85.60 \\
\hline Mountains & 84.72 & 84.48 & 81.04 & 83.20 \\
\hline Food & 85.16 & 85.48 & 85.08 & 84.80 \\
\hline
\end{tabular}

The comparison graph of average accuracy with different similarity measurements is shown in Figure 9. 


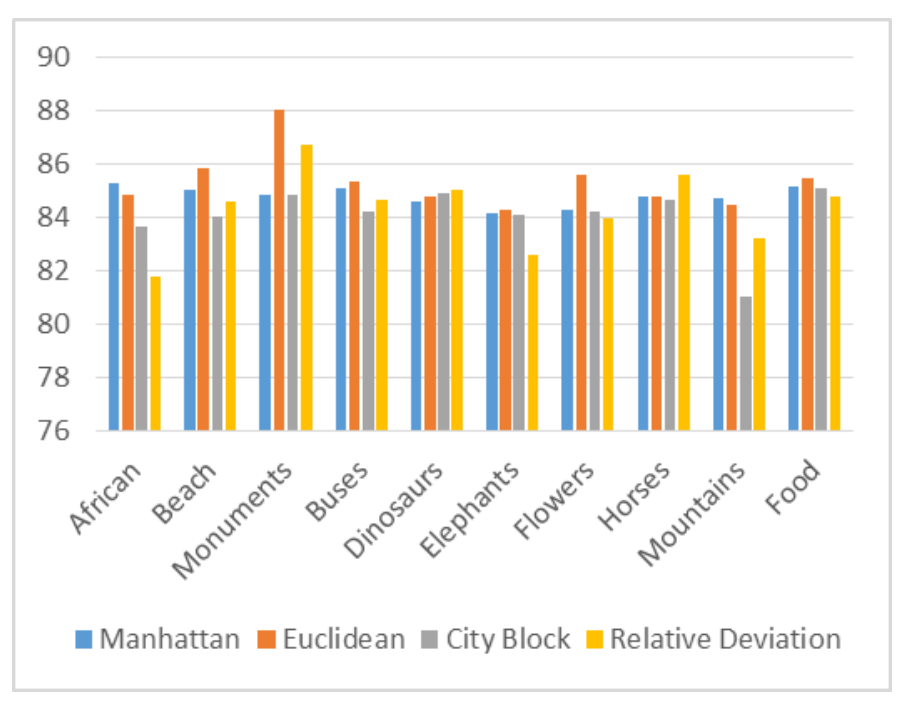

\section{Figure 9. Comparison Graph of Average Accuracy with Different Similarity Measurements}

Table 2 describes the comparative analysis between existing methods and the proposed method. Here, this proposed approach is providing better result over method [22] and method [6]. In Figure 10 and 11, the bar graph of avg. precision and avg. recall is illustrated to perform the comparative analysis among method [22], method [21] and proposed method. From the Table 2 and Figure 10, 11 it is found that our classification based method is performing well in term of precision and recall over existing methods.

Table 2. Comparison Based on Avg. Precision and Avg. Recall

\begin{tabular}{|l|c|c|c|c|c|c|}
\hline \multirow{2}{*}{$\begin{array}{l}\text { Image } \\
\text { category }\end{array}$} & \multicolumn{2}{|c|}{ Method [22] } & \multicolumn{2}{c|}{ Method [6] } & \multicolumn{2}{c|}{ Proposed Result } \\
\cline { 2 - 7 } & $\mathrm{P}$ & $\mathrm{R}$ & $\mathrm{P}$ & $\mathrm{R}$ & $\mathrm{P}$ & $\mathrm{R}$ \\
\hline African & 76.00 & 43.16 & 76.78 & 86 & 77.12 & 73.66 \\
\hline Beach & 58.70 & 32.87 & 82.92 & 68 & 75.16 & 72.20 \\
\hline Monuments & 71.40 & 35.93 & 76.08 & 70 & 80.09 & 78.40 \\
\hline Buses & 96.30 & 69.37 & 74.07 & 80 & 94.16 & 96.16 \\
\hline Dinosaurs & 100.0 & 99.66 & 75.92 & 82 & 100 & 100 \\
\hline Elephants & 74.10 & 35.58 & 85.41 & 82 & 88.25 & 90.25 \\
\hline Flowers & 94.50 & 69.56 & 88.89 & 80 & 97.10 & 96.80 \\
\hline Horses & 94.10 & 62.93 & 75.00 & 78 & 98.16 & 97.33 \\
\hline Mountains & 45.70 & 24.00 & 89.13 & 82 & 82.67 & 84.40 \\
\hline Food & 73.30 & 36.47 & 84.78 & 78 & 86.91 & 83.66 \\
\hline
\end{tabular}




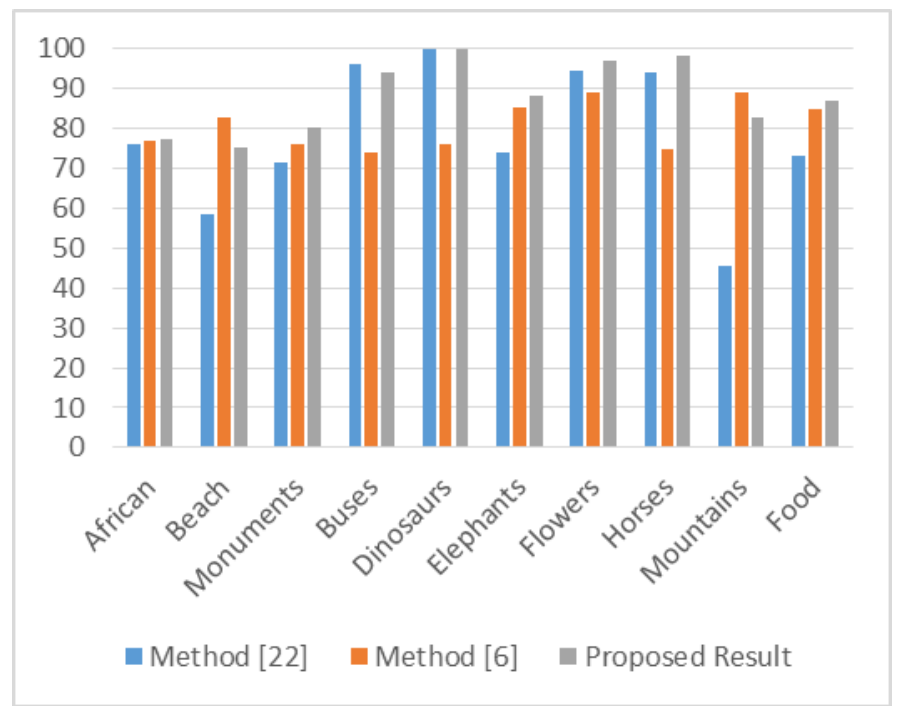

Figure 10. Comparison Graph of Avg. Precision

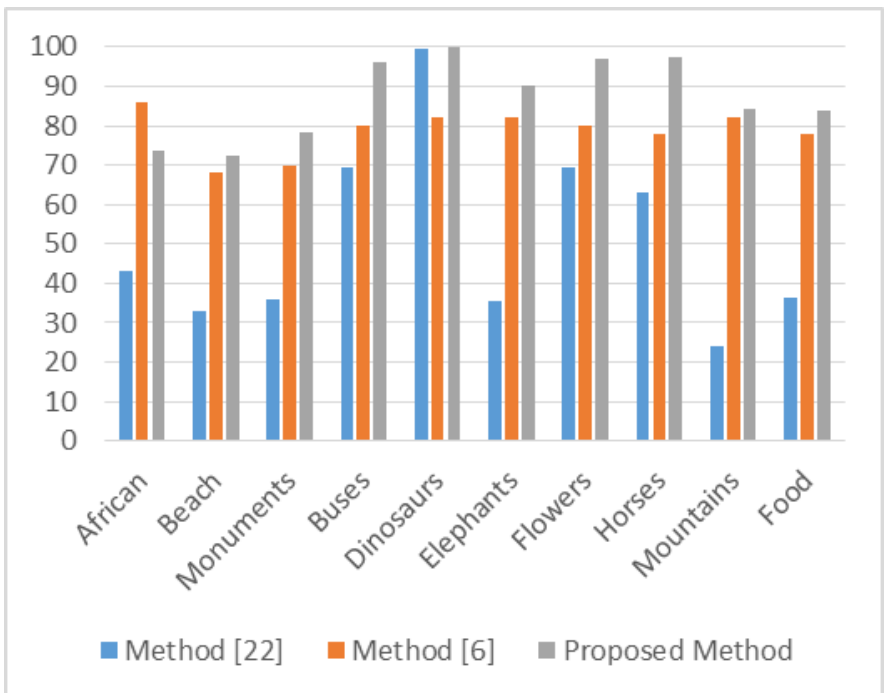

Figure 11. Comparison Graph of Avg. Recall 


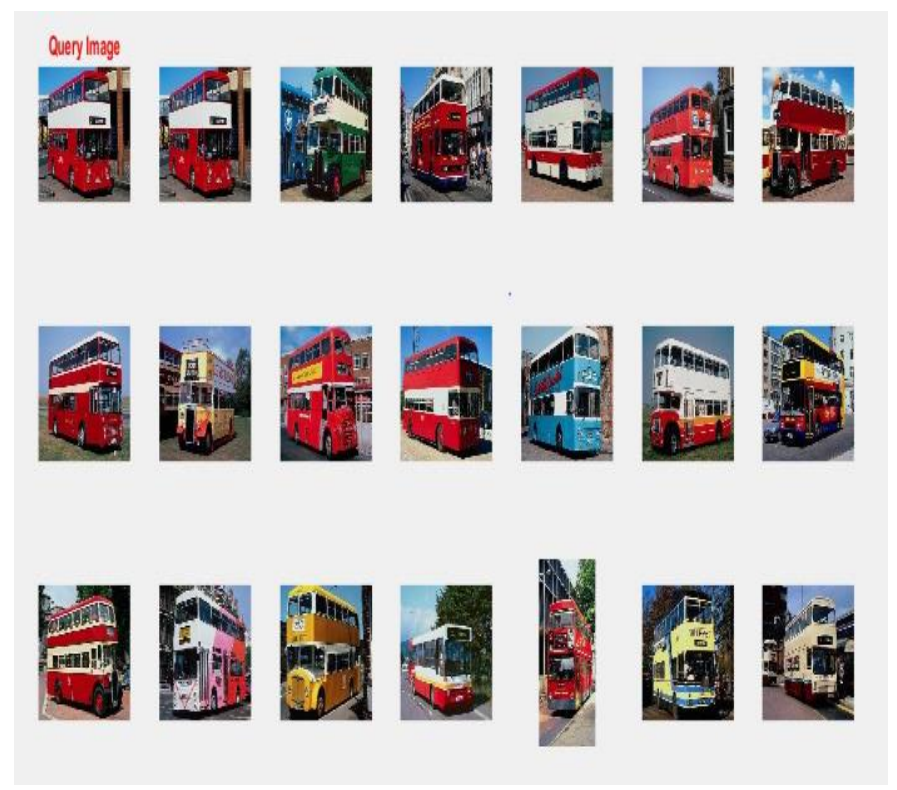

Figure 12. Retrieved Images After Applying SVM for Bus

\section{Conclusion}

In this paper, an approach for content based image retrieval by using color, texture, shape and RBF-SVM classifier has presented. Here as feature extraction, the quantized HSV histogram is used to extract color information of the image. Along with histogram, the discrete wavelet transforms as well as EHD are used to extract the complex texture pattern, spatial as well as edge information of the image. On the fusion of these color, texture and shape feature descriptors, the RBF-SVM classifier is applied to classify the images into different classes accurately by the help of radial basis kernel function. In the experimental analysis, it is found that this proposed approach yields the average accuracy of $85 \%$ with Euclidean distance on the Wang image database. This proposed method is matched with different existing methodologies. It is observed that this method produced much better results than existing schemes of image retrieval. Furthermore, the different kind of machine learning techniques can be applied in place of SVM to make it more efficient.

\section{References}

[1] S. M. H. Khan, A. Hussain, and I. F. T. Alshaikhli, "Comparative Study on Content Based Image Retrieval (CBIR)," Advanced Computer Science Application and Technologies (ACSAT), International Conference on Kuala Lumpur, (2013), pp. 61-66.

[2] K. Ashok Kumar and, Y. V. Bhaskar Reddy, "Content Based Image Retrieval Using SVM Algorithm," International Journal of Electrical and Electronics Engineering (IJEEE), ISSN (PRINT): 2231 - 5284, vol. 1, no. 3, (2012).

[3] C. A. Hussain, D. V. Rao and S. A. Mastani, "Low Level Feature Extration Methods For Content Based Image Retrieval," 978-1-4799-7678-2/15/@2015 IEEE

[4] S. Somnugpong and K. Khiewwan, "Content- Based Image Retrieval Using a Combination of Color Correlograms and Edge Direction Histogram," 978-1-5050-2033-1/16/@2016 IEEE

[5] K. Sugamya, S. Pabboju and A. V. Babu, "A cbir classification using support vector machines," 978-14673-8810-8/16/\$31.00 @2016 IEEE

[6] E. Gupta, and R. S. Kushwah, "Combination of Global and Local Features using DWT with SVM for CBIR," 978-1-4673-7231-2/15/\$31.00 @2015 IEEE

[7] S. Sankar Ganesh, and K. Ramar, "Content Based Image Retrieval Using Heuristic Particle Swarm Optimization," Asian Journal of Information Technology 15 (12), ISSN: 1682-3915, (2016), pp. 19291935

[8] Dr. Ananthi Sheshasaayee, and Jasmine .C, "Relevance Feedback Techniques Implemented in CBIR: Current Trends and Issues," International Journal of Engineering trends and Technology (IJETT)Volume 10 Number 4 - Apr 2014, ISSN: 2231-5381, pp. 166-174 
[9] Vinee. V. Kawade, and Arti. V. Bang, "Content Based Image Retrieval Using Interactive Genetic Algorithm," Annual IEEE India Conference (INDICON), 978-1-4799-5364-6/14/\$31.00 @2014 IEEE

[10] K. Ashok Kumar \&Y.V.Bhaskar Reddy, "Content Based Image Retrieval Using SVM Algorithm," International Journal of Electrical and Electronics Engineering (IJEEE), ISSN (PRINT): 2231 -5284, Vol-1, Iss-3, 2012.

[11] Garima Gupta, and Manish Dixit, "CBIR on Biometric Application using Hough Transform with DCD, DWT Features and SVM Classification," International Journal of Engineering and Innovative Technology, ISSN: 2277-3754, Volume 4, Issue 12, June 2016.

[12] F. Long, H. Zhang, H. Dagan, and D. Feng, "Fundamentals of Content Based Image Retrieval," Multimedia Signal Processing Book, Chapter 1, Springer-Verlag, Berlin Heidelberg New York, 2003.

[13] Rajkumar Jain, and Punit Johari, "An Improved Approach of CBIR using Color Based HSV Quantization and Shape Based Edge Detection Algorithm," 978-1-5090-0774-5/16 C2016 IEEE

[14] Garima Gupta, and Manish Dixit, "CBIR on Biometric Application using Hough Transform with DCD, DWT Features and SVM Classification," International Journal of Engineering and Innovative Technology, ISSN: 2277-3754, Volume 4, Issue 12, June 2016.

[15] A. Haris Rangkuti, Nashrul Hakiem, Rizal Broer Bahaweres, Agus Harjoko, and Agfianto Eko Putro, "Analysis of Image Similarity with CBIR Concept Using Wavelet Transform and Threshold Algorithm," 978-1-4799-0210-1/13@2013 IEEE

[16] C.S. Won, D.K. Park, and S.J. Park, "Efficient Use of Edge Histogram Descriptor-An Overview," ETRI Journal, vol. 24, Number 1, Feb 2002, pp. 23-30.

[17] Pradnya Vikhar, and Pravin Karde, "Improved CBIR System using Edge Histogram Descriptor (EHD) and Support Vector Machine (SVM)", 978-1-5090-5515-9/16/\$31.00 @2016 IEEE

[18] Sujata T Bhairnallykar, V.B.Gaikwad, “ Content based Medical Image Retrieval with SVM Classification and Relevance Feedback", International Journal of Applied Information Systems (IJAIS) - ISSN : 2249-0868, PP 25-29, 2013

[19] N. Upadhyaya, and M. Dixit, "An Insight to Various CBIR Technique: A Survey,” International Journal of Advanced Research in Computer Science and Software Engineering, ISSN: 2277 128X, vol. 6, no. 5, (2016).

[20] N. Upadhyaya and M. Dixit, "A Review: Relating Low Level Features to High Level Semantics in CBIR," International Journal of Signal Processing, Image Processing and Pattern Recognition", vol. 9, no. 3, (2016), pp. 433-444.

[21] E. Paul, and A. S. A. Beegom, "Mining Images for Images Annotation using SURF Detection Technique," International Confrence on Control, Communication \& Computing India (ICCC), 978-14649-7349-4/15//\$31.00 @2015 IEEE

[22] R. Gali, M. L. Dewal, and R. S. Anand, "Genetic Algorithm for Content Based Image Retrieval," 978-07695-4821-0/12 \$26.00 @ 2012 IEEE, DOI 10.1109/CICSyN.2012.52

[23] D. Kurchaniya, and P. K. Johary, "Analysis of Different Similarity Measures in Image Retrieval Based on Texture and Shape," International Research Journal of Engineering and Technology (IRJET), eISSN: 2395 -0056, p-ISSN: 2395-0072, vol. 4 no. 4 (2017). 
International Journal of Signal Processing, Image Processing and Pattern Recognition Vol. 10, No. 9 (2017) 